OPEN ACCESS

Edited by:

Dirk Balmer,

Syngenta Crop Protection, LLC,

Switzerland

Reviewed by:

Ming Wang,

University of California, Riverside,

United States

Zonghua Wang

Fujian Agriculture and Forestry

University, China

*Correspondence:

Wenjun Zhu

82862108@qq.com

Wei Wei

weiwei1985723@163.com

Specialty section:

This article was submitted to Plant Microbe Interactions,

a section of the journal

Frontiers in Microbiology

Received: 27 April 2017 Accepted: 12 July 2017

Published: 25 July 2017

Citation:

Zhu W, Zhou M, Xiong Z, Peng F and

Wei W (2017) The CAMP-PKA

Signaling Pathway Regulates

Pathogenicity, Hyphal Growth, Appressorial Formation, Conidiation, and Stress Tolerance in Colletotrichum

higginsianum

Front. Microbiol. 8:1416. doi: 10.3389/fmicb.2017.01416

\section{The cAMP-PKA Signaling Pathway Regulates Pathogenicity, Hyphal Growth, Appressorial Formation, Conidiation, and Stress Tolerance in Colletotrichum higginsianum}

\author{
Wenjun Zhu ${ }^{1 *}$, Man Zhou ${ }^{2}$, Zeyang Xiong ${ }^{2}$, Fang Peng ${ }^{1}$ and Wei Wei ${ }^{2 *}$ \\ ${ }^{1}$ College of Biology and Pharmaceutical Engineering, Wuhan Polytechnic University, Wuhan, China, ${ }^{2}$ Institute for \\ Interdisciplinary Research, Jianghan University, Wuhan, China
}

Colletotrichum higginsianum is an economically important pathogen that causes anthracnose disease in a wide range of cruciferous crops. Understanding the mechanisms of the cruciferous plant-C. higginsianum interactions will be important in facilitating efficient control of anthracnose diseases. The cAMP-PKA signaling pathway plays important roles in diverse physiological processes of multiple pathogens. C. higginsianum contains two genes, ChPKA1 and ChPKA2, that encode the catalytic subunits of cyclic AMP (CAMP)-dependent protein kinase A (PKA). To analyze the role of cAMP signaling pathway in pathogenicity and development in $C$. higginsianum, we characterized ChPKA1 and ChPKA2 genes, and adenylate cyclase (ChAC) gene. The ChPKA1 and ChAC deletion mutants were unable to cause disease and significantly reduced in hyphal growth, tolerance to cell wall inhibitors, conidiation, and appressorial formation with abnormal germ tubes, but they had an increased tolerance to elevated temperatures and exogenous $\mathrm{H}_{2} \mathrm{O}_{2}$. In contrast, the ChPKA2 mutant had no detectable alteration of phenotypes, suggesting that ChPKA1 contributes mainly to PKA activities in C. higginsianum. Moreover, we failed to generate $\triangle$ ChPKA1ChPKA2 double mutant, indicating that deletion of both PKA catalytic subunits is lethal in C. higginsianum and the two catalytic subunits possibly have overlapping functions. These results indicated that ChPKA1 is the major PKA catalytic subunit in CAMP-PKA signaling pathway and plays significant roles in hyphal growth, pathogenicity, appressorial formation, conidiation, and stress tolerance in C. higginsianum.

\footnotetext{
Keywords: Colletotrichum higginsianum, cAMP-PKA signaling pathway, ChPKA1, pathogenicity, appressorial formation, stress tolerance
}

\section{INTRODUCTION}

The hemibiotrophic fungus Colletotrichum higginsianum is an economically important pathogen that causes anthracnose disease on a wide range of cruciferous plants, including Brassica, Raphanus, and Arabidopsis thaliana (Narusaka et al., 2004; Yang et al., 2008; Hyde et al., 2009). To invade plant tissue successfully, $C$. higginsianum conidia germinate and form the melanized infection structure 
appressorium at the tips of conidial germ tubes after attaching to plant surfaces. After that, C. higginsianum penetrates the cuticle and plant cell wall using high turgor pressure generated in melanized appressoria for further infection (O'Connell et al., 2012).The morphogenesis and invasion of several fungal pathogens are dependent on recognition and transduction of host environmental stimuli and surface cues to its downstream via multiple signal transduction pathways, especially the cyclic adenosine monophosphate (cAMP)-PKA signaling pathway (Kronstad et al., 2011; Li et al., 2012). Protein kinase A (PKA) is a serine/threonine protein kinase that serves as the main intracellular target of cAMP, a key secondary messenger synthesized by adenylate cyclase (AC). PKA consists of two catalytic and two regulatory subunits; binding of cAMP to regulatory subunits leads to the activation of catalytic subunits, which phosphorylate downstream target proteins and regulate a variety of physiological processes (Robertson and Fink, 1998).

To date, the cAMP-PKA signaling pathway is known to function in a range of physiological processes in fungi, including growth, cell wall integrity, cell differentiation, stress responses, pathogenicity, colonization, and secondary metabolism (Lengeler et al., 2000; Fillinger et al., 2002; Oliver et al., 2002; Xue et al., 2008; Turrà et al., 2014). In soilborne fungal pathogen Fusarium oxysporum, deletion of cAMP-dependent PKA (FoCPKA) led to failure to penetrate into the vascular system of $A$. thaliana roots, loss of virulence, and reduced vegetative growth and spore production (Kim et al., 2011). In F. verticillioides, the cAMP-PKA pathway is involved in mycelia growth, conidiation, bikaverin production, and plant infection (Choi and $\mathrm{Xu}$, 2010). In F. graminearum, functional studies of the FAC1 (adenylate cyclase), CPK1 and CPK2 genes have demonstrated that the cAMP-PKA signaling pathway plays significant roles in morphogenetic switch, growth, deoxynivalenol (DON) production, pathogenicity, and sexual reproduction (Bormann et al., 2014; Hu et al., 2014). In Ustilago maydis, the cAMPPKA signaling pathway is important for dimorphic switching and mating (Durrenberger et al., 1998). In Magnaporthe oryzae, the cAMP-PKA signaling pathway is involved in surface recognition, asexual, and pathogenic differentiation (Ramanujam and Naqvi, 2010). The Cyclase-associated protein Cap1 from M. oryzae is involved in activation of adenylate cyclase, appressorium morphogenesis, and plant infection (Zhou et al., 2012). Deletion of PKA catalytic subunit gene $C p k 1$ and adenylate cyclase gene $C A C 1$ in C. lagenarium caused defect in conidia germination and pathogenicity, attenuated growth rate and reduced conidiation (Yamauchi et al., 2004). In C. orbiculare, Ras GTPase activating protein CoIral contributes to infection-related morphogenesis by regulating CAMP and MAPK signaling pathways (Harata and Kubo, 2014). The AreA transcription factor from F. graminearum was shown to mediate the regulation of DON synthesis by cAMP signaling (Hou et al., 2015). Recently, it was reported that cAMP signaling pathway is involved in the regulation of DON biosynthesis by two pathway-specific transcription factors TRI (trichothecene biosynthesis) 6 and TRI10 (Jiang et al., 2016). Therefore, these findings suggest significant roles for cAMPPKA signaling pathway in multiple physiological processes of different microorganisms, especially during the steps of surface recognition and penetration which are critical in the infection cycle of many plant pathogenic fungi. Inhibition on the cAMPPKA signaling pathway of pathogens will disturb infection progress and facilitate efficient control of crop diseases.

Although many studies have been done to elaborate cAMPPKA signaling pathway in other fungi, the specific roles of cAMP-PKA signaling for infection-related morphogenesis and infectious growth remain largely unknown in C. higginsianum. Because the cAMP-PKA signaling pathway contributes to multiple physiological processes of fungal pathogens, especially surface recognition and penetration (Li et al., 2012), characterization of C. higginsianum cAMP-PKA signaling will help elucidate the mechanism of the C. higginsianumcruciferous crops interaction and facilitate the efficient control of anthracnose disease.

In this study, to better understand the cAMP-PKA signaling pathway in $C$. higginsianum, we investigated the functions of PKA catalytic subunits ChPKA1 (CH063_00098) and ChPKA2 (CH063_12956), and adenylate cyclase ChAC (CH063_06008). Whereas, $\triangle$ ChPKA2 mutant had no obviously detectable phenotypes, the $\triangle C h P K A 1$ and $\triangle C h A C$ mutants had similar phenotypes with pleiotropic defects in hyphal growth, appressorial formation, stress tolerance, conidiation, and pathogenicity. Application of exogenous cAMP could partially rescue the phenotype defects, indicating that ChPKA1 is the major PKA catalytic subunit in $C$. higginsianum. Moreover, although the conidia of $\triangle C h P K A 1$ and $\triangle C h A C$ mutants had defects in appressorial formation at high conidial density $\left(10^{6}\right.$ conidia/ml), they partially differentiated into appressoria at low conidial density $\left(10^{4}\right.$ conidia/ml $)$ with much longer germ tubes compared with that of wild type strain. In addition, we failed to obtain $\triangle C h P K A 1 \triangle C h P K A 2$ double mutant, indicating that deletion of both PKA catalytic subunit genes is lethal in C. higginsianum. Overall, our data indicate that cAMP-PKA signaling pathway plays essential roles in hyphal growth, stress tolerance, conidiation, appressorial formation, and pathogenicity in C. higginsianum.

\section{MATERIALS AND METHODS}

\section{Strains and Plant Materials}

C. higginsianum wild-type strain IMI349061 (CH-1) was cultured on potato dextrose agar (PDA) at $25^{\circ} \mathrm{C}$ and stored in PDA slants at $4^{\circ} \mathrm{C}$ for further use. The wild type plants of A. thaliana Col-0 used in this study for pathogenicity test were grown in greenhouse at $20 \pm 2{ }^{\circ} \mathrm{C}$, under a $12 \mathrm{~h}$ light/dark cycle. Agrobacterium tumefaciems strain EHA105 was used for transformation of C. higginsianum and Escherichia coli strain JM109 was used to propagate all plasmids.

\section{Bioinformatics Data and Programs Used in This Study}

The publicly available genomic sequence database of C. higginsianum at JGI (http://genome.jgi.doe.gov/Colhi1/ Colhil.home.html) was used to characterize ChPKA1 (CH063_00098), ChPKA2 (CH063_12956), and ChAC (CH063_06008) genes. BLAST analysis was done by using 
NCBI (http://www.ncbi.nlm.nih.gov/) and UniProt (http://www. uniprot.org/blast/). MEGA program was used for the production of phylogenetic tree with unrooted neighbor-joining method. Domains identification was performed by using SMART MODE (http://smart.embl-heidelberg.de/smart/change_mode.pl). The Clustal $X$ program was used for amino acid alignments.

\section{Manipulation of Nucleic Acids}

The genomic DNA of C. higginsianum wild type strain and other derivative transformants were extracted using genomic DNA purification kit (Axygen, USA) according to the manufacturer's protocols. Southern blot analysis was performed as previously described (Liu et al., 2013). The $20 \mu \mathrm{g}$ genomic DNA of each strain was digested overnight, then size-fractionated through a $0.8 \%$ agarose gel and mounted on positively charged nylon membrane. The nylon membrane was then hybridized with a probe amplified by primers (Supplementary Table S1) and labeled with digoxigenin (DIG)-dUTP using the PCR DIG Probe Synthesis Kit (Roche, Mannheim, Germany) according to the manufacturer's protocol.

Total RNA was isolated with TriZOL reagent (Invitrogen, Carlsbad, USA) according to manufacturer's instructions and then stored at $-80^{\circ} \mathrm{C}$ for further studies. The total RNA samples were treated with DNase I (RNase Free) (Takara, Dalian, China) at $37^{\circ} \mathrm{C}$ for $30 \mathrm{~min}$ and used to generate the first strand cDNA with the High Capacity cDNA Reverse Transcription Kit (Applied Biosystems ${ }^{\mathrm{TM}}$, Foster, CA, USA) according to manufacturer's instructions. The first strand CDNA was stored at $-20^{\circ} \mathrm{C}$ for further studies. Genes expression was analyzed by quantitative real-time reverse polymerase chain reaction (qRTPCR) using a Bio-Rad CFX96 (Bio-Rad, California, USA) and SYBR Premix Ex Taq II (TAKARA, Dalian, China), according to the manufacturer's instructions. The C. higginsianum $\beta$-tubulin gene (CH063_04743) was used to normalize the RNA sample for each round of qRT-PCR. The PCR conditions were as follows: denaturation at $95^{\circ} \mathrm{C}$ for $3 \mathrm{~min} ; 40$ cycles of $95^{\circ} \mathrm{C}$ for $15 \mathrm{~s}$, $55^{\circ} \mathrm{C}$ for $20 \mathrm{~s}$ and $72^{\circ} \mathrm{C}$ for $25 \mathrm{~s}$; final step of $72^{\circ} \mathrm{C}$ for $10 \mathrm{~min}$. Primer pairs for qRT-PCR reactions were designed either across or flanking an intron and listed in Supplementary Table S1. For each gene, $\mathrm{qRT}$-PCR assays were repeated at least twice, with each repetition having three independent replicates.

\section{Gene Replacement and Complementation}

To characterize ChPKA1, ChPKA2, and ChAC genes, the genes replacement vectors pChPKA1-3300, pChPKA2-3300, and pChAC-3300 were generated as described (Ma et al., 2017). The $5^{\prime}$ (about $500 \mathrm{bp}$ )- and $3^{\prime}$ (about $500 \mathrm{bp}$ )- flanks of the ORF of each genes were amplified from genomic DNA of the wild type strain $\mathrm{CH}-1$ by $\mathrm{PCR}$ with primer pairs (Supplementary Table S1). The $5^{\prime}$ - and $3^{\prime}$ - fragments of each genes were then cloned into the upstream and downstream of hph cassette respectively, using Gibson Assembly Master Mix kit (New England Biolabs, Massachusetts, USA) according to the manufacturer's instructions. Then, the $5^{\prime}$ fragment- $h p h-3^{\prime}$ fragment cassettes of each gene were cloned into pNeoP3300 (Wei et al., 2016), resulting in gene replacement vectors, which had the selective marker $h p h$ gene flanked by the ORF flanking sequences from each of the genes (Supplementary Figure S1A).
The gene complementation vectors pChPKA1-Com, pChPKA2-Com and pChAC-Com were constructed that the cDNA of each gene was amplified by RT-PCR with primers (Supplementary Table S1) and cloned into the same sites of pCIT vector, which contained the constitutive $\mathrm{P} \operatorname{trp} C$ promoter and $\mathrm{T} \operatorname{trp} C$ terminator. Finally, the $\mathrm{P} \operatorname{trpC} \mathrm{C}-\mathrm{cDNA}-\mathrm{T} \operatorname{trp} C$ cassette was cloned into pNeoP3300, resulting in complementation vector.

Agrobacterium-mediated transformation was performed as previously described (Wei et al., 2016). Transformants were transferred to PDA plates containing $50 \mu \mathrm{g} / \mathrm{ml}$ of hygromycin B (Roche, Mannheim, Germany) or $150 \mu \mathrm{g} / \mathrm{ml} \mathrm{G418} \mathrm{(Ameresco,}$ $\mathrm{OH}$, USA) for a second round of selection and further confirmed by Southern blot and qRT-PCR analysis.

\section{Phenotype Analysis}

The growth rates of all the transformants and the wild-type strain were assayed as previously described (Wei et al., 2016). All strains were initially grown on PDA for 7 days. The mycelial agar discs were then taken from the active colony edge, inoculated into the center of the PDA petri dish at $25^{\circ} \mathrm{C}$, and then the colony diameters from 1 to $7 \mathrm{dpi}$ were examined. The colony morphology and conidiation of all strains were examined after being grown on PDA plates at $25^{\circ} \mathrm{C}$ for 14 and 7 days, respectively.

Pathogenicity assays of $C$. higginsianum on Arabidopsis were performed as previously described (Yuan et al., 2016). The conidia harvested from 5-days-old PDA agar cultures at $25^{\circ} \mathrm{C}$ were washed with sterile distilled water twice. The leaf surfaces of each pot of 4-weeks-old Arabidopsis were sprayed with $1 \mathrm{ml}$ conidial suspensions $\left(10^{6}\right.$ conidia/ml). The infected Arabidopsis plants were incubated in humid chambers at $25^{\circ} \mathrm{C}$ for 6 days, and the symptoms were then observed. The experiment was repeated three times, and each strain infected three $A$. thaliana plants each time.

Appressorial formation was measured. $10 \mu \mathrm{l}$ conidial suspensions $\left(10^{6}\right.$ conidia/ml for high conidial density and $10^{4}$ conidia/ml for low conidial density) were spotted on hydrophobic coverslips and incubated in darkness at $25^{\circ} \mathrm{C}$ for $24 \mathrm{~h}$. The percentage of appressorial formation was determined by microscopic examination for at least 200 conidia or appressoria using Nikon Eclipse 80i microscope (Nikon, Tokyo, Japan), under bright-field model using $40 \times$ fold magnification. Each test was repeated at least three times.

The tolerance of transformants and wild-type strain to multiple stress was performed. The mycelial plugs from the active colony edge were inoculated on PDA containing $300 \mu \mathrm{g} / \mathrm{ml}$ Calcofluor White (CFW), $10 \mathrm{mM} \mathrm{H}_{2} \mathrm{O}_{2}$, and $300 \mu \mathrm{g} / \mathrm{ml}$ Congo Red (CR) respectively and PDA media as controls. The growth inhibition rate (\%) of stress to the transformants and wild type strain was analyzed by measuring the colony diameters as previously described (Wei et al., 2016).

The tolerance of these strains to high temperature was performed by inoculating mycelial plugs from the active colony edge of each strain on PDA and then culturing at 25 and $35^{\circ} \mathrm{C}$ respectively for 6 days. The phenotypes and growth inhibition rate of these strains were then analyzed. 


\section{Statistical Analysis}

The data were analyzed with Origin 7.5 (OriginLab Corporation, Massachusetts, USA) using ANOVA (one-way, $P \leq 0.01$ ). Results of all graphs represent the mean value \pm SD. Asterisks and different letters in the graphs indicate statistical differences, $P \leq 0.01$.

\section{RESULTS}

\section{Bioinformatics Analysis of ChPKA1 and ChPKA2}

The C. higginsianum genome contains two PKA catalytic subunit genes, CH063_00098 and CH063_12956, that were respectively named ChPKA1 and ChPKA2. ChPKA1 encodes a 507-amino-acids protein and ChPKA2 encodes a 392amino-acids protein. The SMART MODE (http://smart. embl-heidelberg.de/smart/change_mode.pl) analysis result indicates that both of ChPKA1 and ChPKA2 contain a Serine/Threonine protein kinase catalytic (S_TKc) domain and an Extension to Ser/Thr-type protein kinase (S_TK_X) domain (Supplementary Figure S2A), which plays a key role in catalysis of protein phosphorylation. BLAST searches of fungal genomes with ChPKA1 and ChPKA2 showed the presence of homologs in a large number of fungi. Multiple sequence alignment and phylogenetic analysis revealed significant sequence conservation (Supplementary Figures S2B-D).

\section{Up-Regulation of ChPKA1 and ChAC Expression but not ChPKA2 during Appressorial Formation}

To gain insight into the functions of ChPKA1, ChPKA2, and $C h A C$, we firstly examined the gene expression patterns during different infection stages of $C$. higginsianum by qRT-PCR as previous described (Liu et al., 2013). The result demonstrated that the expression levels of ChPKA1 and ChAC but not ChPKA2, were significantly up-regulated during conidia germinating (5 hpi) and appressorial formation (22 hpi) stages (Figure 1), indicating that ChPKA1 and ChAC may play significant roles in conidia germinating and appressorial formation of C. higginsianum.

\section{ChPKA1 and ChAC but not ChPKA2 Are Indispensable for Growth, Colony Phenotype, and Conidiation}

To assess the function of ChPKA1 and ChPKA2 in C. higginsianum, the ChPKA1 and ChPKA2 deletion mutants were generated and screened by growing on PDA containing hygromycin and further confirmed by Southern blot and qRTPCR (Supplementary Figures S1B,C). Whereas the ChPKA2 deletion mutant $\triangle$ ChPKA2 had no detectable phenotype variation compared with wild type strain, the ChPKA1 deletion mutant $\triangle C h P K A 1$ showed a significantly reduced growth rate (Figures 2A, 3; Supplementary Table S2), and the $\triangle C h P K A 1$ sectored on PDA with obvious dark color and abnormal colony (Figure 2A). Compared with $\triangle C h P K A 2$ and wild type strain, the $\triangle C h P K A 1$ also showed obvious reduction of conidiation
(Figure 4; Supplementary Table S2). For complementation assays, the complementary vector pChPKA1-Com, pChPKA2Com, and pChAC-Com were reintroduced into mutants to generate complementary strains ChPKA1-Com, ChPKA2-Com, and $C h A C$-Com. Defects in hyphal growth, colony morphology, and conidiation were rescued in ChPKA1-Com and ChAC-Com strains (Figures 2A， 3，4; Supplementary Table S2). These results indicated that deletion of ChPKA1 but not ChPKA2 is directly responsible for the phenotype defects in $\triangle$ ChPKA1, and ChPKA1 is involved in growth, colony phenotype and conidiation in C. higginsianum.

In addition, as adenylate cyclase (AC) contributes to the synthesis of cAMP and plays a significant role in cAMPPKA signaling pathway, we also functionally analyzed ChAC by gene replacement of ChAC (CH063_06008) adenylate cyclase gene to generate ChAC knockout mutant $\triangle C h A C$ (Supplementary Figure S1). Compared to wild type strain, the growth rate and conidiation of $\triangle C h A C$ were obviously reduced on PDA medium (Figures 3, 4), and the $\triangle C h A C$ also showed an albino colony after 6 days incubation on PDA (Figures 7A,B). Furthermore, in the presence of exogenous $10 \mathrm{mM}$ cAMP in PDA medium, the growth rate and conidiation in the $\triangle C h A C$, but not $\triangle C h P K A 1$, mutant were restored to the levels of the wild type strain (Figures 3,4). These results further suggested the importance of cAMP-PKA signaling pathway in the regulation of growth and conidiation in C. higginsianum.

Totally, we had obtained at least five individual mutant strains and three individual complementary strains for each gene, and all the mutants and complementary strains of each gene showed the same phenotype (Supplementary Table S2). Thus, we selected two mutants and one complementary strain of each gene for Southern blot, and choose one mutant and one complementary strain of each gene for further phenotype analysis.

\section{ChPKA1 and ChAC Are Important for Pathogenicity and Appressorial Formation}

To further assess the function of ChPKAl in pathogenicity, the infection assays against the Arabidopsis were performed as described above. The results demonstrated that the wild type, complementary strains and $\triangle$ ChPKA2 could cause typically water-soaked and dark necrotic lesions on leaves, whereas the $\triangle C h P K A 1$ and $\triangle C h A C$ failed to cause obvious disease symptoms on Arabidopsis leaves after inoculating for 6 days (Figure 2B), which is similar to previous study in which the PKA and $A C$ mutants of $C$. lagenarium did not cause any obvious lesions on cucumber leaves, even inoculated on wounded leaves (Yamauchi et al., 2004). These results indicated that ChPKA1 and ChAC are required for pathogenicity in C. higginsianum.

The appressorial formation of each strain was also analyzed. The results suggested that at high conidial density $\left(10^{6}\right.$ conidia/ml), most conidia ( $>65 \%$ ) of wild type, complementary strains and $\triangle C h P K A 2$ could form melanized appressoria on hydrophobic surface within $24 \mathrm{~h}$ (Figures 5A, 6A). In contrast, most $\triangle$ ChPKA1 and $\triangle C h A C$ conidia $(>97 \%)$ did not form appressoria within $24 \mathrm{~h}$. They showed obvious defects in appressorial formation and developed poorly $(<3 \%)$ on 

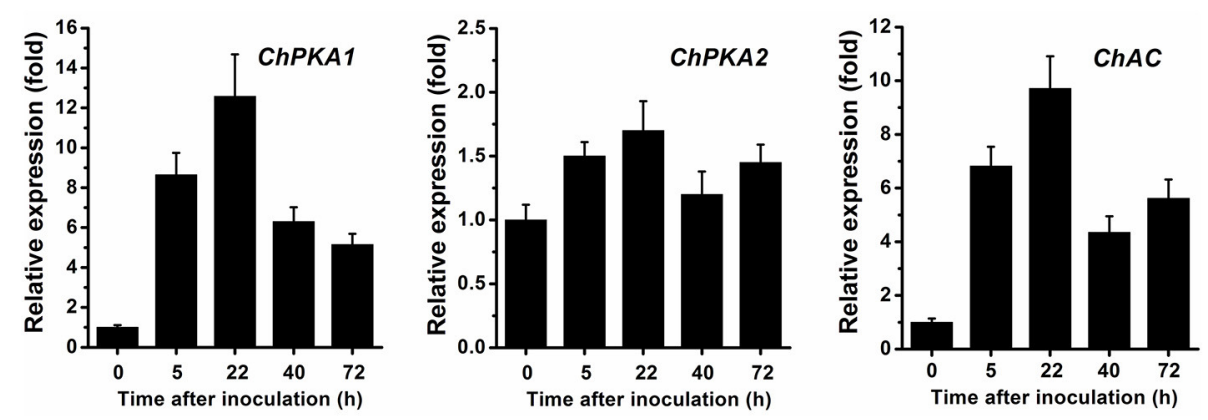

FIGURE 1 | Expression patterns of ChPKA1, ChPKA2, and ChAC during infection stages were evaluated by qRT-PCR. RNA was extracted from infected Arabidopsis seedlings at 0 hpi, 5 hpi (conidial germination), 22 hpi (appressorial formation), 40 hpi (biotrophic infection stage), and 72 hpi (necrotrophic infection stage), respectively. The genes expression of $C$. higginsianum conidia inoculated on Arabidopsis at $0 \mathrm{~h}$ was set as level 1 and relative levels of transcript were calculated using the comparative Ct method. Transcript level of the C. higginsianum $\beta$-tubulin gene (CH063_04743) was used to normalize different samples. Data represent means and standard deviations of three independent replications.

A

$\mathrm{CH}-1$

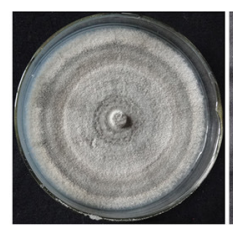

B

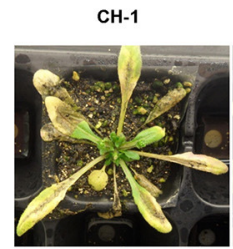

$\triangle$ ChPKA1

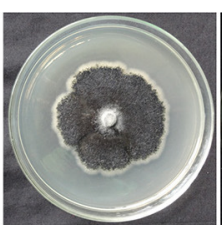

ChPKA1-Com

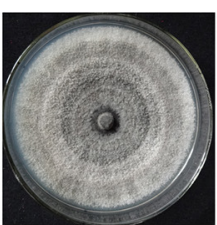

ChPKA1-Com

$\triangle$ ChPKA1

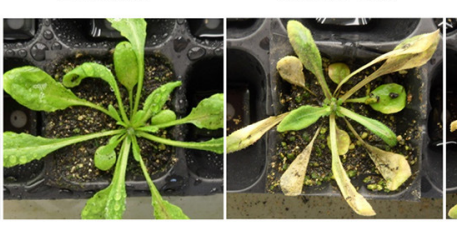

$\triangle C h P K A 2$

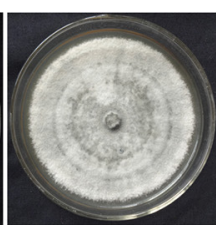

$\triangle$ ChPKA2

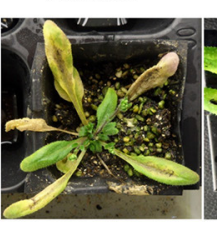

ChPKA2-Com

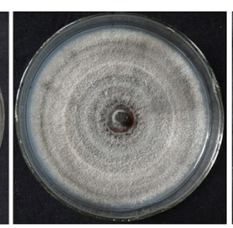

ChPKA2-Com

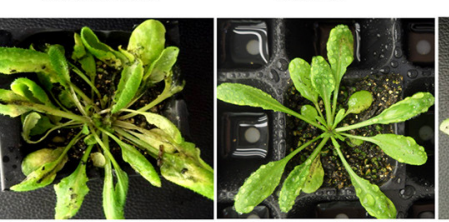

$\triangle \mathrm{ChAC}$

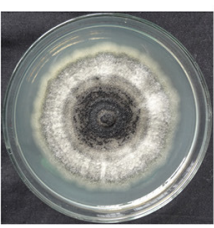

$\triangle \mathrm{ChAC}$

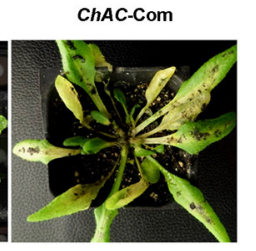

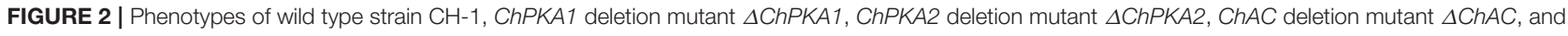
complementary strains ChPKA1-Com, ChPKA2-Com and ChAC-Com. (A) Colony morphology of indicated strains. These strains were grown on PDA medium at $25^{\circ} \mathrm{C}$ for 14 days. (B) Pathogenicity assays of indicated strains. 4-weeks-old $A$. thaliana were respectively sprayed with $1 \mathrm{ml}$ conidial suspensions (10 6 conidia/ml) of indicated strains and the infected Arabidopsis plants were incubated in darkness and humid chambers at $25^{\circ} \mathrm{C}$ for 6 days, and then the symptoms were observed.

hydrophobic coverslips (Figures 5A, 6A). The appressorium formation rates of $\triangle C h P K A 1$ and $\triangle C h A C$ were significantly lower than the rate of wild type strain $\mathrm{CH}-1$ and complementary strains (Figure 6A). Interestingly, the appressorial formation defect in $\triangle$ ChPKA1 and $\triangle$ ChAC could be partially rescued by incubation of conidia at low density $\left(10^{4}\right.$ conidia/ml), but the appressorial formation rates were still much lower than the rate of wild type strain (Figure 6A). Although conidia of $\triangle C h P K A 1$ and $\triangle C h A C$ could form appressoria at low density $\left(10^{4}\right.$ conidia/ml), they developed much longer germ tubes compared with that of the wild type strain $\mathrm{CH}-1$ (Figure 5B). In addition, application of exogenous cAMP could partly restore the size of the germ tube of $\triangle C h A C$ mutant but not $\triangle C h P K A 1$ (Figure 5B), and exogenous cAMP could also significantly increase the appressorial formation rate in $\triangle C h A C$ but not $\triangle C h P K A 1$ (Figures 6B,C). These results indicated that ChPKA1 and $C h A C$ play significant roles in regulation of appressorial formation, thus to affect the pathogenicity of $C$. higginsianum.

\section{cAMP-PKA Signaling Pathway Is Involved in Stress Tolerance}

To further examine whether the ChPKAl and ChAC are involved in stress tolerance, the growth of wild type strain, $\triangle C h P K A 1, \triangle C h P K A 2$, and $\triangle C h A C$ were measured on PDA plates supplemented with various compounds including $300 \mu \mathrm{g} / \mathrm{ml}$ Calcofluor White (CFW), $10 \mathrm{mM} \mathrm{H}_{2} \mathrm{O}_{2}$, and $300 \mu \mathrm{g} / \mathrm{ml} \mathrm{CR}$ respectively. The tolerance of these strains to high temperature $\left(35^{\circ} \mathrm{C}\right)$ was also determined. The results demonstrated that on PDA media with CFW or CR, inhibition of growth rate of $\triangle C h P K A 1$ and $\triangle C h A C$ was obviously 


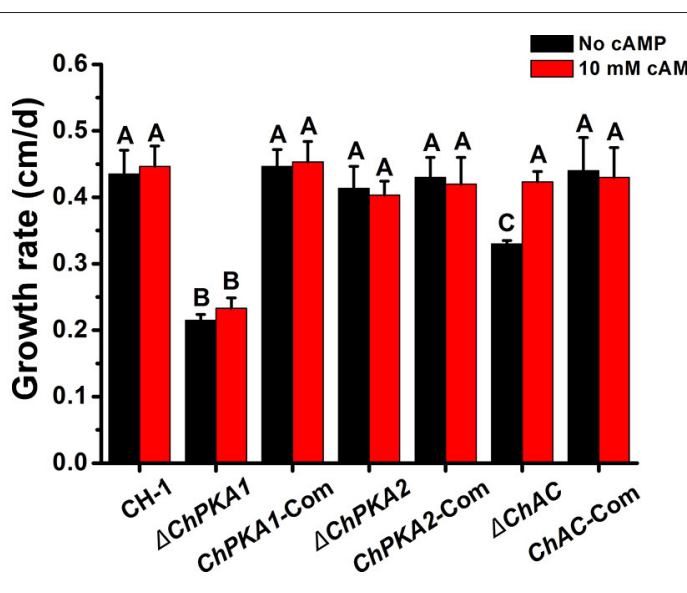

FIGURE 3 | Growth rate of indicated strains. Growth rate of indicated strains cultured on PDA medium with (red) or without (black) exogenous $10 \mathrm{mM}$ CAMP at $25^{\circ} \mathrm{C}$. Bars represent standard deviations of three replications. Different letters in the graph indicate statistical differences, $P \leq 0.01$.

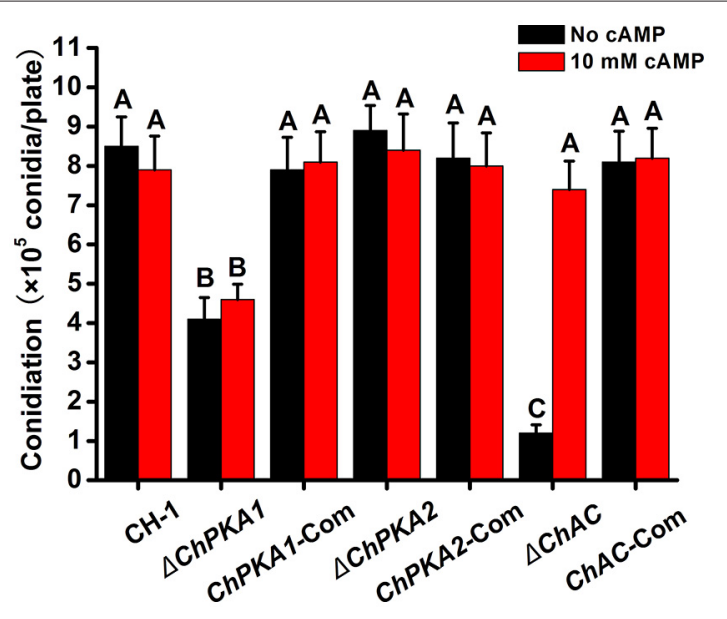

FIGURE 4 | Conidial production of indicated strains. Conidiation of indicated strains cultured on PDA plates with (red) or without (black) exogenous $10 \mathrm{mM}$ CAMP at $25^{\circ} \mathrm{C}$ for 7 days. Conidiation of each strain was determined by collecting conidia with a hematocytometer. Bars represent standard deviations of three replicates. Different letters in the graph indicate statistical differences, $P \leq 0.01$.

higher than that of wild type strain and $\triangle C h P K A 2$ mutant (Figures 7A,C), indicating that ChPKAl and ChAC may function to maintain the fungal cell wall integrity by positive regulation.

In contrast, the $\triangle C h P K A 1$ and $\triangle C h A C$ mutants showed higher tolerance to $10 \mathrm{mM} \mathrm{H}_{2} \mathrm{O}_{2}$ and high temperature $\left(35^{\circ} \mathrm{C}\right)$ compared with wild type strain (Figures $7 \mathbf{B}, \mathbf{D}$ ), which is similar with that of its homolog in F. verticillioides (Choi and Xu, 2010), suggesting that ChPKA1 and ChAC may negatively regulate $\mathrm{H}_{2} \mathrm{O}_{2}$ and high temperature tolerance in C. higginsianum. Moreover, previous studies reported that the GSY2 and HSP26 genes are up-regulated under heat stress condition in yeast
(Schnell et al., 1992; Gasch et al., 2000), and the expression levels of FvGSY2 and FvHSP26 genes of F. verticillioides are higher in adenylate cyclase mutant after heat treatment (Choi and $\mathrm{Xu}$, 2010). Similar results were also reported that the PKA mutant of F. graminearum was more tolerant to heat stress than the wild type strain for hyphal growth, and the relative expression levels of FgHSP70 and FgGSY2 in PKA mutant were much higher than in wild type strain when cultured at $35^{\circ} \mathrm{C}$ (Hu et al., 2014). In our study, we speculated that the homolog genes of GSY2 and HSP70 in C. higginsianum may be also involved in the high temperature tolerance of $\triangle C h P K A 1$ and $\triangle C h A C$ mutants. In order to verify this assumption, we identified the homolog of GSY2 in C. higginsianum, named ChGSY2 (CH063_00792), and homolog of HSP70, named ChHSP70 (CH063_01329). Then, we comparatively analyzed the expression levels of ChGSY2 and ChHSP70 in the hyphae of $\triangle C h P K A 1, \triangle C h A C$, and wild type strain after incubating at $35^{\circ} \mathrm{C}$ for $3 \mathrm{~h}$. The result demonstrated that the expression levels of ChGSY2 and ChHSP70 were obviously up-regulated in $\triangle C h P K A 1$ and $\triangle C h A C$ compared with that of the expression in wild type strain (Figure 8), indicating that ChPKA1 and ChAC may negatively regulate the expression of some stress response genes under high temperature condition in C. higginsianum.

\section{DISCUSSION}

The cAMP-PKA signaling pathway plays significant and conserved roles in mediating cellular processes, including growth, conidial formation, stress tolerance, and pathogenesis in many fungal pathogens (Turrà et al., 2014; Han et al., 2015; Martin-Urdiroz et al., 2016). However, the roles of cAMPPKA signaling pathway in anthracnose fungus C. higginsianum remain uncharacterized. In this study, we elucidated the functions of ChPKA1, ChPKA2, and ChAC in pathogenic and physiological development of C. higginsianum, mainly including growth, conidiation, stress tolerance, appressorial formation, and pathogenicity. Our findings demonstrated that ChPKA1 and ChAC, but not ChPKA2, are essential for growth, appressorial formation, conidiation, and pathogenicity in C. higginsianum.

Normally, filamentous fungi have two PKA catalytic subunits genes, but only one of them contributes to the major function of PKA activities. Deletion of the major PKA catalytic subunit gene causes obvious reduction in growth rate and conidiation, whereas the other one plays only a minor role or has no detectable functions (Shimizu and Keller, 2001; Lee et al., 2003; Banno et al., 2005; Ni et al., 2005; Schumacher et al., 2008; Choi and Xu, 2010; Hu et al., 2014). In our study, targeted deletion of ChPKA1 resulted in significant reduction in growth rate (Figure 3 ) and conidiation (Figure 4), and sectored colony with obvious dark color (Figure 2A), whereas deletion of ChPKA2 did not caused any obvious phenotype variation compared with wild type strain. In addition, we also analyzed the phenotype of adenylate cyclase mutant $\triangle C h A C$, which showed similar reduction in growth rate (Figure 3) and conidiation (Figure 4). Application of exogenous cAMP could partially rescued the phenotype defects of $\triangle C h A C$ (Figures 3, 4). These results indicated that ChPKA1 is the major 
A

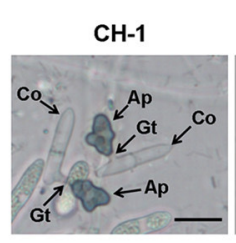

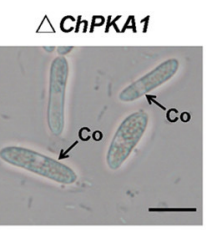
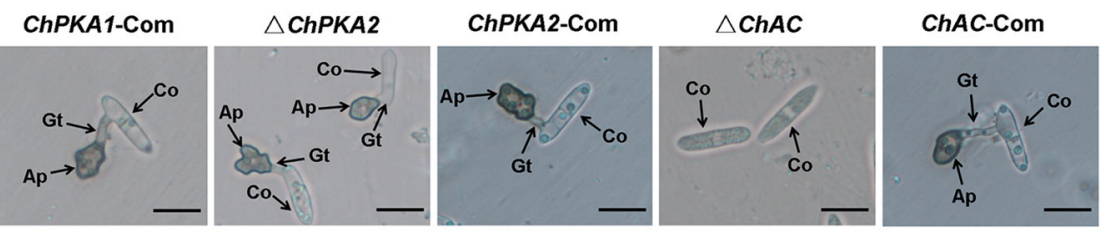

B

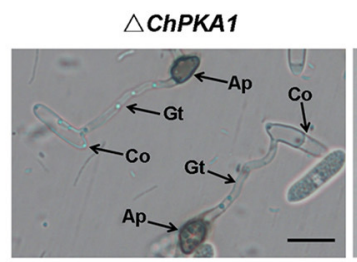

$\triangle C h P K A 1+10 \mathrm{mM}$ CAMP
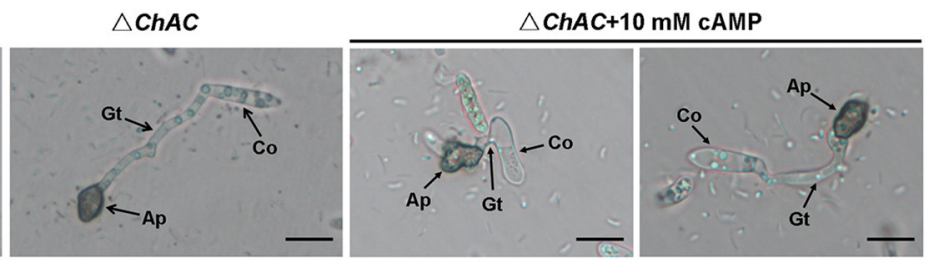

FIGURE 5 | The appressorial development of indicated strains on hydrophobic coverslips. (A) The development of appressoria on plastic microscopic coverslips at $25^{\circ} \mathrm{C}$ for $24 \mathrm{~h}$. Conidial density was $10^{6} \mathrm{conidia} / \mathrm{ml}$. (B) Appressorial formation on plastic microscopic coverslips at $25^{\circ} \mathrm{C}$ for $24 \mathrm{~h}$, with or without exogenous $10 \mathrm{mM}$ cAMP. Conidial density was $10^{4}$ conidia/ml. The images were taken by using Nikon Eclipse 80i microscope (Nikon, Tokyo, Japan), under bright-field model with $40 \times$ fold magnification. Ap, appressoria; Co, conidia; Gt, germ tubes. Bars $=10 \mu \mathrm{m}$.
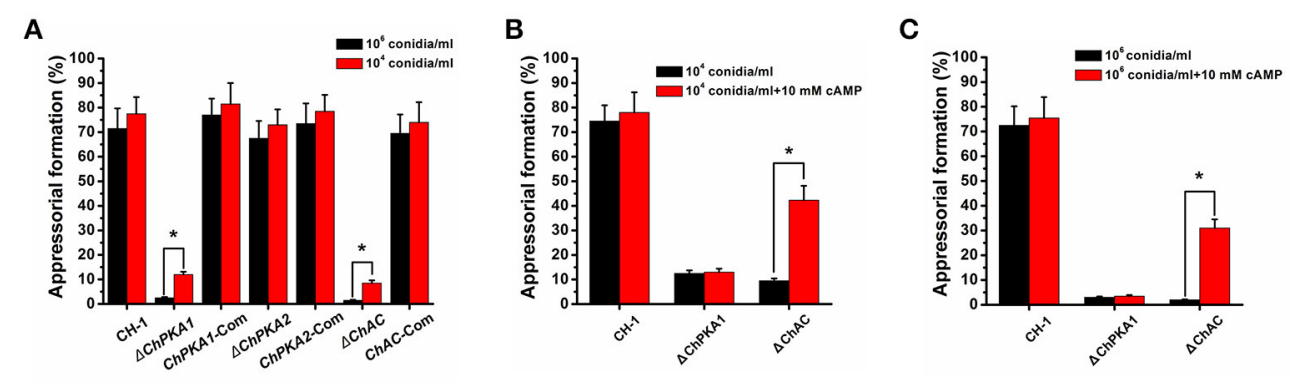

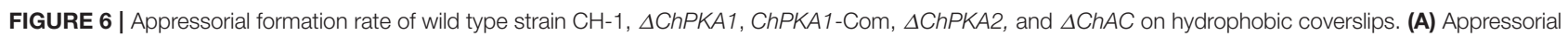
formation rate of indicated strains at different conidial density $\left(10^{6}\right.$ and $10^{4} \mathrm{conidia} / \mathrm{ml}$, respectively) on hydrophobic coverslips at $25^{\circ} \mathrm{C}$ for $24 \mathrm{~h}$. (B) Appressorial formation rate of $\mathrm{CH}-1, \triangle \mathrm{ChPKA1}$, and $\triangle \mathrm{ChAC}$ at $10^{4}$ conidia/ml with or without exogenous $10 \mathrm{mM} \mathrm{CAMP}$ on hydrophobic coverslips at $25^{\circ} \mathrm{C}$ for $24 \mathrm{~h}$. (C) Appressorial formation rate of $\mathrm{CH}-1, \triangle \mathrm{ChPKA1}$, and $\triangle \mathrm{ChAC}$ at $10^{6}$ conidia/ml with or without exogenous $10 \mathrm{mM} \mathrm{cAMP}$ on hydrophobic coverslips at $25^{\circ} \mathrm{C}$ for $24 \mathrm{~h}$. Asterisks in the graph indicate statistical differences, $P \leq 0.01$.

PKA catalytic subunit in the cAMP-PKA signaling pathway of C. higginsianum.

In Aspergillus flavus, F. verticillioides, S. sclerotiorum, F. graminearum, C. lagenarium, and F. oxysporum, deletion of the adenylate cyclase or PKA gene resulted in significant reduction in growth rate (Yamauchi et al., 2004; Jurick and Rollins, 2007; Choi and Xu, 2010; Pei-Bao et al., 2010; Kim et al., 2011; Hu et al., 2014; Yang et al., 2016), whereas the opposite was observed in $M$. oryzae (Mitchell and Dean, 1995; Choi and Dean, 1997; Adachi and Hamer, 1998). In our study, the growth rate of deletion mutants $\triangle C h P K A 1$ and $\triangle C h A C$ were obviously decreased compared to wild type strain (Figure 3), supporting the diverse roles of cAMP-PKA signaling pathway in different plant fungal pathogens.

Appressorium functions as an important infection structure and plays a critical role in primary penetration The cAMP signaling pathway regulates the appressorial formation in several pathogens. In $M$. oryzae, the feedback between the cAMP-PKA and MAPK signaling pathways regulates appressorium morphogenesis and plant infection (Zhou et al., 2012, 2014). In C. lagenarium, cAMP-PKA signaling pathway cooperated with MAPK to regulate appressorial formation and infectious growth (Takano et al., 2000; Yamauchi et al., 2004). Ras GTPase activating protein CoIral from C. orbiculare was shown to be involved in infection-related morphogenesis and pathogenicity by proper regulation of cAMP and MAPK signaling pathways through CoRas2 (Harata and Kubo, 2014). In our study, deletion of ChPKA1 and ChAC caused obvious defect in appressorial formation at high conidial density $\left(10^{6}\right.$ conidia/ml). Although defection of appressorial formation in mutants $\triangle C h P K A 1$ and $\triangle C h A C$ could be partially rescued at low conidial density $\left(10^{4}\right.$ conidia/ml) (Figures 5A, 6A), conidia of mutants developed much longer germ tubes when compared with that of wild type strain (Figure 5B). Furthermore, the pathogenicity of mutants $\triangle C h P K A 1$ and $\triangle C h A C$ on A. thaliana was also defective (Figure 2B), inferring that defective virulence was possibly caused by the abnormal appressoria formation. In summary, we conclude that cAMP-PKA signaling pathway 

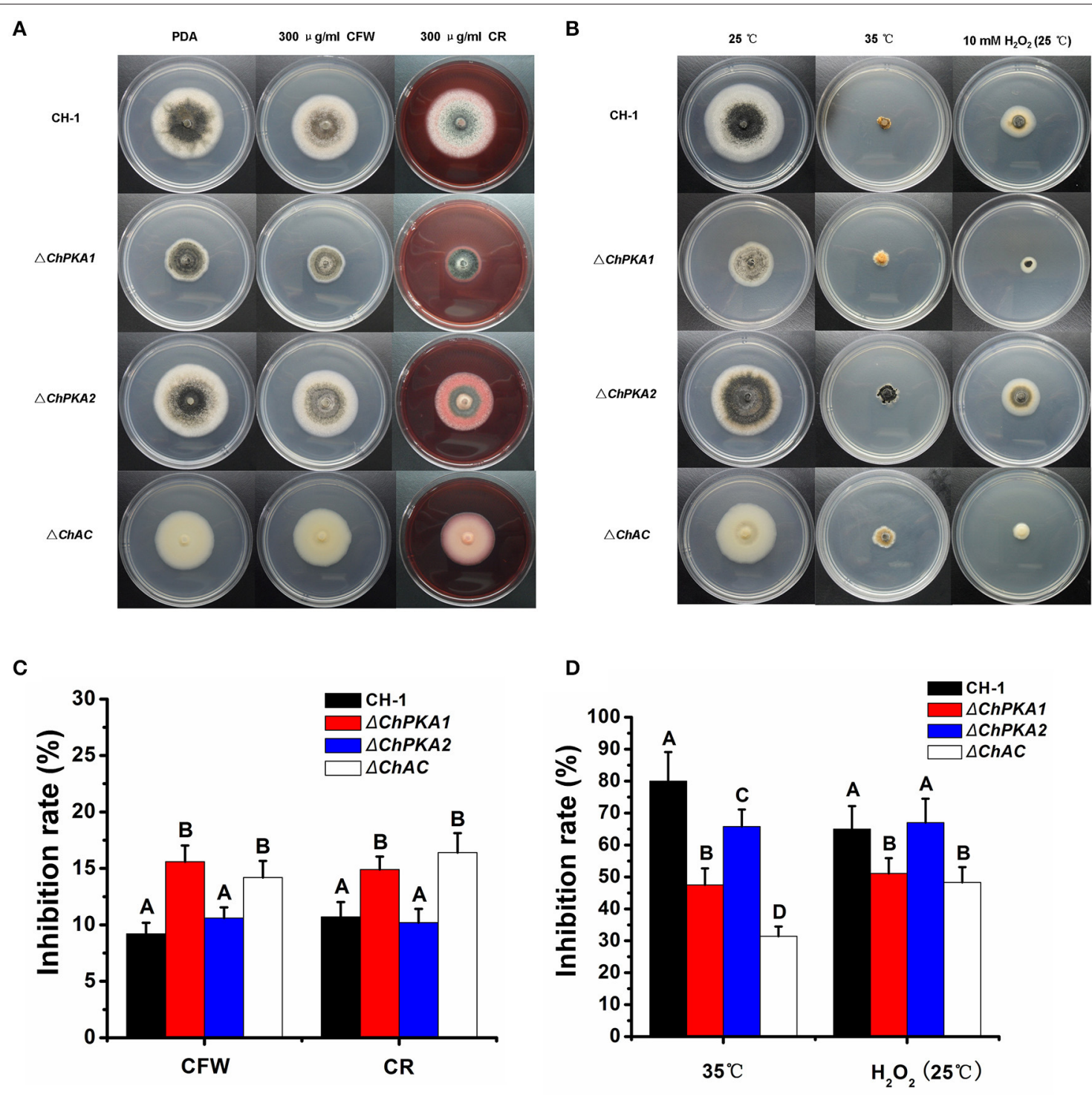

FIGURE 7 | Stress tolerance assays of wild type strain $\mathrm{CH}-1, \triangle C h P K A 1, \triangle C h P K A 2$, and $\triangle C h A C$. (A) $\mathrm{CH}-1, \triangle C h P K A 1, \triangle C h P K A 2$, and $\triangle C h A C$ were treated with

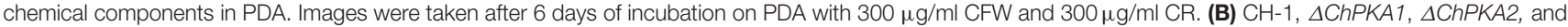
$\triangle C h A C$ cultured on PDA at different temperature or with $10 \mathrm{mM} \mathrm{H}_{2} \mathrm{O}_{2}$. Images were taken after 6 days of incubation at 25 and $35^{\circ} \mathrm{C}$, or with $10 \mathrm{mM} \mathrm{H} \mathrm{O}_{2}$, respectively. (C) Inhibition rate of the radiated growth of $\mathrm{CH}-1, \triangle \mathrm{ChPKA1}, \triangle \mathrm{ChPKA2}$, and $\triangle \mathrm{ChAC}$ on the PDA with chemical components. (D) Inhibition rate of the radiated growth of $\mathrm{CH}-1, \triangle \mathrm{ChPKA1}, \triangle \mathrm{ChPKA2}$, and $\triangle \mathrm{ChAC}$ on the PDA at $35^{\circ} \mathrm{C}$ compared with that of their growth at $25^{\circ} \mathrm{C}$ or with $10 \mathrm{mM} \mathrm{H}_{2} \mathrm{O}_{2} \cdot \mathrm{Means}$ and standard deviations were calculated from three replicates. Different letters in the graph indicate statistical differences, $P \leq 0.01$.

regulates appressorial formation, thus to affect C. higginsianum pathogenicity.

Recently, it was reported that intracellular cAMP level in the vegetative hyphae of C. gloeosporioides was controlled by Rho GTPases CgRhoB and regulated conidia germination and stress tolerance ( $\mathrm{Xu}$ et al., 2016). The Ras guanine-nucleotide exchange factor protein ChRgf acted as an important modulator upstream of several cAMP-PKA signaling pathway to regulate vegetative growth, conidiation, infection-related structure development, and stress responses of C. higginsianum ( $\mathrm{Gu}$ et al., 2017). Our work also examined whether the stress tolerance was affected in cAMP-PKA pathway mutants. The results indicated that the $\triangle C h P K A 1$ and $\triangle C h A C$ mutants are more sensitive to cell wall inhibitors $\mathrm{CFW}$ and $\mathrm{CR}$ compared to wild type strain and $\triangle C h P K A 2$ mutant (Figures 7A,C). Interestingly, in contrast, the $\triangle C h P K A 1$ and $\triangle C h A C$ mutants showed increased tolerance to elevated temperatures and exogenous $\mathrm{H}_{2} \mathrm{O}_{2}$ when compared with wild type strain (Figures 7B,D). In addition, the expression levels of ChHSP70 and ChGSY2 in $\triangle C h P K A 1$ and $\triangle C h A C$ mutants were higher than in wild type strain when cultured at $35^{\circ} \mathrm{C}$ (Figure 8). These results are similar with previous report that, as well as increased expression 


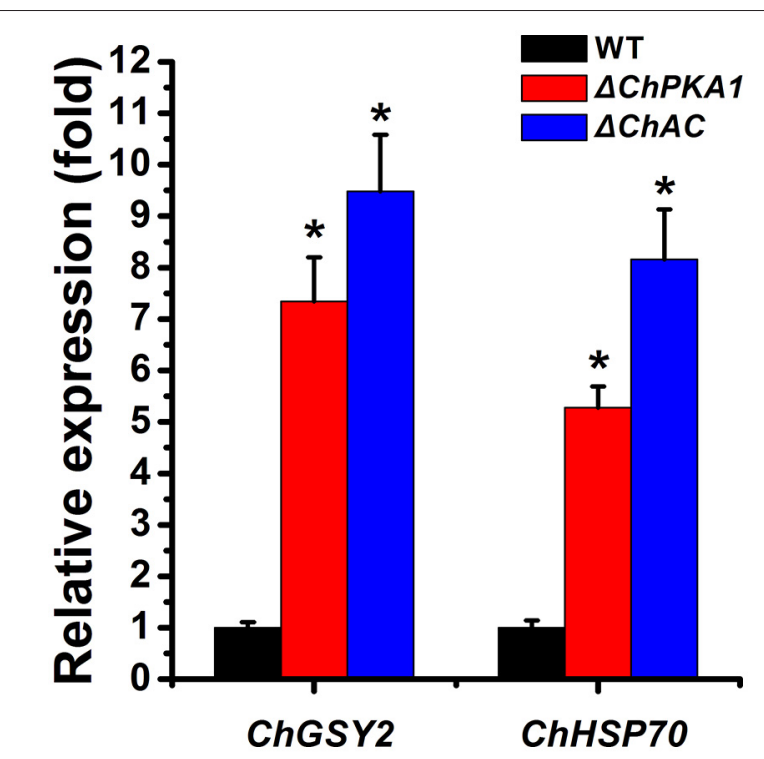

FIGURE 8 | The relative expression of ChGSY2 and ChHSP70 in the hyphae of wild type strain $\mathrm{CH}-1$, mutants $\triangle \mathrm{ChPKA1}$ and $\triangle \mathrm{ChAC}$ incubated at $35^{\circ} \mathrm{C}$ for $3 \mathrm{~h}$. The relative expression of target genes in $\mathrm{CH}-1$ was set as level 1 . Expression level of $C$. higginsianum $\beta$-tubulin (CH063_04743) gene was used to normalize different samples. Bars represent standard deviations from three replications. Asterisks indicate statistical differences from $\triangle C h P K A 1$ and $\triangle C h A C, P \leq 0.01$.

level of HSP70 and ChGSY2, the cAMP-PKA pathway mutants are more tolerant to heat stress than wild type strain in F. verticillioides and F. graminearum (Choi and Xu, 2010; Hu et al., 2014).

Furthermore, in this study, we have also tried several times to generate $\triangle$ ChPKA1ChPKA2 double mutant, but failed, indicating that deletion of both PKA catalytic subunits (ChPKA1 and ChPKA2) is lethal in C. higginsianum, which is similar with that of homologs in A. nidulans (Ni et al., 2005) and M. oryzae (Choi and $\mathrm{Xu}, 2010$ ). However, this finding is opposite to that of homolog in F. graminearum (Hu et al., 2014), suggesting the functional diversity of PKA among diverse fungal species. It also indicated that ChPKA1 and ChPKA2 may have overlapping functions.

Briefly, in this study, we found that cAMP-PKA signaling pathway is involved in appressorium formation, pathogenicity, growth rate, conidiation, and stress tolerance in C. higginsianum. Given these properties, targeting cAMP-PKA signaling pathway could be a good strategy to control cruciferous crop anthracnose diseases. The results described above will also enhance our understanding of the interaction mechanisms between C. higginsianum and A. thaliana.

\section{CONCLUSION}

ChPKA1 was experimentally confirmed to be the major PKA catalytic subunit in CAMP-PKA signaling pathway and essential to hyphal growth, pathogenicity, appressorial formation, conidiation, and stress tolerance in C. higginsianum, indicating that ChPKA1 plays diverse and essential roles in this fungal pathogen.

\section{AUTHOR CONTRIBUTIONS}

Conceived and designed the experiments: WZ and WW. Performed the experiments: WZ and WW. Analyzed the experiment data: WZ, MZ, ZX, FP, and WW. Contributed reagents/materials/analysis tools: $\mathrm{WZ}, \mathrm{FP}$, and $\mathrm{WW}$. Wrote the paper: WZ and WW. All authors read and approved the final manuscript.

\section{ACKNOWLEDGMENTS}

The research was financially supported by the Hubei Province Department of Education Scientific Research Plan Guidance Project (B2016297). We thank reviewers for their kind suggestions.

\section{SUPPLEMENTARY MATERIAL}

The Supplementary Material for this article can be found online at: http://journal.frontiersin.org/article/10.3389/fmicb. 2017.01416/full\#supplementary-material

Supplementary Figure S1 | Construction of replacement vectors and confirmation of genes mutants. (A) Strategic map of ChPKA1, ChPKA2, and $C h A C$ replacement construct. The hygromycin phosphotransferase ( $h p h)$ gene cassette was cloned into the corresponding sites to replace the ORFs of ChPKA1, ChPKA2, and ChAC genes. P1, P2, and P3 indicated the hybridization probes. (B) Southern blot analysis of mutants. Genomic DNA (2 $\mu \mathrm{g}$ per lane) of $\triangle$ ChPKA1 and ChPKA1-Com were digested with Kpnl, genomic DNA (20 $\mathrm{gg}$ per lane) of $\triangle C h P K A 2$ and $\triangle C h A C$ were digested with Sacl. The nylon membranes with genomic DNA of ChPKA1, ChPKA2 and ChAC were respectively hybridized with a probe corresponding to P1, P2, and P3. Genomic DNA (20 $\mu \mathrm{g}$ per lane) of wild type strain $\mathrm{CH}-1$ was used as control. (C) The relative expression of ChPKA1 in $\triangle$ ChPKA1 and ChPKA1-Com, ChPKA2 in $\triangle C h P K A 2$, and ChAC in $\triangle C h A C$. The relative expression of target genes in $\mathrm{CH}-1$ was set as level 1. Expression level of $\beta$-tubulin (CH063_04743) gene was used to normalize different samples. Bars represent standard deviations from three replications. ND, not detected.

Supplementary Figure $\mathbf{S} 2$ | Characterization of the C. higginsianum ChPKA1 and ChPKA2. (A) The domain structures of C. higginsianum ChPKA1 and ChPKA2 as annotated by SMART MODE (http://smart.embl-heidelberg.de/ smart/change_mode.pl). (B) Alignment analysis of the amino acid sequences of ChPKA1 and its homologs from other fungi using Clustal X program. MO1: M. oryzae CPKA (MGG_06368, E-value: 0.0, 68\% identity); FG1: F. graminearum CPK1 (FGSG_07251, E-value: 0.0, 92\% identity); NC1: Neurospora crassa PKAC-1 (NCU06240, E-value: 0.0, 70\% identity); BC1: Botrytis cinerea PKA1 (BC1G_10410, E-value: 0.0, 68\% identity); CL1: C. lagenarium CPK1 (AB127956.1, E-value: 0.0, 90\% identity); VD1: Verticillium dahliae (VDAG_06474, E-value: 0.0, 69\% identity); SS1: Sclerotinia sclerotiorum (SS1G_13577, E-value: 0.0, 68\% identity). (C) Alignment analysis of the amino acid sequences of ChPKA2 and its homologs from other fungi using Clustal X program. VD2: V. dahliae (VDAG_02641, E-value: 0.0, 77\% identity); FG2: F. graminearum CPK2 (FGSG_08729, E-value: 5.2E-171, 77.7\% identity); MO2: M. oryzae CPKB (MGG_02832, E-value: 0.0, 70\% identity); NC2: N. crassa PKAC-2 (NCU00682, E-value: 0.0, 70\% identity); BC2: B. cinerea PKA2 (BC1G_07542, E-value: 0.0, 74\% identity); SS2: S. sclerotiorum (SS1G_03171, E-value: 0.0, 72\% identity); CG2: C. gloeosporioides (AFA28258, E-value: 0.0, 90\% identity). (D) Phylogenetic analysis of ChPKA1 and ChPKA2 of C. higginsianum and their homologs from other fungi. The 
amino acid sequences were analyzed by MEGA version 4 with Unrooted Neighbor-joining algorithm. Bootstrap values were calculated from 1,000 bootstrap replicates. Only bootstrap support values $>50 \%$ are shown. The black circle indicates ChPKA1; black triangle indicates ChPKA2.

Supplementary Table S1 | Primers used for vector construction and PCR.

\section{REFERENCES}

Adachi, K., and Hamer, J. E. (1998). Divergent cAMP signaling pathways regulate growth and pathogenesis in the rice blast fungus Magnaporthe grisea. Plant Cell 10, 1361-1373. doi: $10.1105 /$ tpc. 10.8 .1361

Banno, S., Ochiai, N., Noguchi, R., Kimura, M., Yamaguchi, I., Kanzaki, S., et al. (2005). A catalytic subunit of cyclic AMP-dependent protein kinase, PKAC1, regulates asexual differentiation in Neurospora crassa. Genes Genet. Syst. 80, 25-34. doi: 10.1266/ggs. 80.25

Bormann, J., Boenisch, M. J., Brückner, E., Firat, D., and Schäfer, W. (2014). The adenylyl cyclase plays a regulatory role in the morphogenetic switch from vegetative to pathogenic lifestyle of Fusarium graminearum on wheat. PLoS ONE 9:e91135. doi: 10.1371/journal.pone.0091135

Choi, W. B., and Dean, R. A. (1997). The adenylate cyclase gene MAC1 of Magnaporthe grisea controls appressorium formation and other aspects of growth and development. Plant Cell 9, 1973-1983. doi: 10.1105/tpc.9.11.1973

Choi, Y. E., and Xu, J. R. (2010). The cAMP signaling pathway in Fusarium verticillioides is important for conidiation, plant infection, and stress responses but not fumonisin production. Mol. Plant Microbe Interact. 23, 522-533. doi: 10.1094/MPMI-23-4-0522

Durrenberger, F., Wong, K., and Kronstad, J. W. (1998). Identification of a cAMP-dependent protein kinase catalytic subunit required for virulence and morphogenesis in Ustilago maydis. Proc. Natl. Acad. Sci. U.S.A. 95, 5684-5689. doi: 10.1073/pnas.95.10.5684

Fillinger, S., Chaveroche, M. K., Shimizu, K., Keller, N., and D'Enfert, C. (2002). cAMP and ras signalling independently control spore germination in the filamentous fungus Aspergillus nidulans. Mol. Microbiol. 44, 1001-1016. doi: 10.1046/j.1365-2958.2002.02933.x

Gasch, A. P., Spellman, P. T., Kao, C. M., Carmel-Harel, O., Eisen, M. B., Storz, G., et al. (2000). Genome expression programs in the response of yeast cells to environmental change. Mol. Biol. Cell 11, 4241-4257. doi: $10.1091 / \mathrm{mbc} .11 .12 .4241$

Gu, Q., Chen, M., Huang, J., Wei, Y. D., Hsiang, T., and Zheng, L. (2017). Multifaceted roles of the ras guanine-nucleotide exchange factor ChRgf in development, pathogenesis, and stress responses of Colletotrichum higginsianum. Phytopathology 107, 433-443. doi: 10.1094/PHYTO-03-16-0137-R

Han, J. H., Lee, H. M., Shin, J. H., Lee, Y. H., and Kim, K. S. (2015). Role of the MoYAK1 protein kinase gene in Magnaporthe oryzae development and pathogenicity. Environ. Microbiol. 17, 4672-4689. doi: 10.1111/1462-2920.13010

Harata, K., and Kubo, Y. (2014). Ras GTPase activating protein Coiral is involved in infection-related morphogenesis by regulating cAMP and MAPK signaling pathways through CoRas2 in Colletotrichum orbiculare. PLoS ONE 9:e109045. doi: 10.1371/journal.pone.0109045

Hou, R., Jiang, C., Zheng, Q., Wang, C., and Xu, J. R. (2015). The area transcription factor mediates the regulation of deoxynivalenol (DON) synthesis by ammonium and cyclic adenosine monophosphate (cAMP) signalling in Fusarium graminearum. Mol. Plant Pathol. 16, 987-999. doi: $10.1111 / \mathrm{mpp} .12254$

Hu, S., Zhou, X., Gu, X., Cao, S., Wang, C., and Xu, J. R. (2014). The cAMP-PKA pathway regulates growth, sexual and asexual differentiation, and pathogenesis in Fusarium graminearum. Mol. Plant Microbe Interact. 27, 557-566. doi: 10.1094/MPMI-10-13-0306-R

Hyde, K. D., Cai, L., Cannon, P. F., Crouch, J. A., Crous, P. W., Damm, U., et al. (2009). Colletotrichum names in current use. Fungal Divers. 39, 147-182. doi: 10.1094/PHYTO-11-13-0315-IA

Jiang, C., Zhang, C., Wu, C., Sun, P., Hou, R., Liu, H., et al. (2016). TRI6 and TRI10 play different roles in the regulation of DON production by
Supplementary Table S2 I Comparison of growth rate, conidial production, and pathogenicity among mutants, complementary strains and the wild type strain $\mathrm{CH}-1$ of $\mathrm{C}$. higginsianum. Means and standard deviations were calculated from three replicates. Different letters in the graph indicate statistical differences, $P \leq 0.01$

cAMP signaling in Fusarium graminearum. Environ. Microbiol. 18, 3689-3701. doi: 10.1111/1462-2920.13279

Jurick, W. M., and Rollins, J. A. (2007). Deletion of the adenylate cyclase (sac1) gene affects multiple developmental pathways and pathogenicity in Sclerotinia sclerotiorum. Fungal Genet. Biol. 44, 521-530. doi: 10.1016/j.fgb.2006.11.005

Kim, H. S., Park, S. Y., Lee, S., Adams, E. L., Czymmek, K., and Kang, S. (2011). Loss of cAMP-dependent protein kinase a affects multiple traits important for root pathogenesis by Fusarium oxysporum. Mol. Plant Microbe Interact. 24, 719-732. doi: 10.1094/MPMI-11-10-0267

Kronstad, J. W., Hu, G., and Choi, J. (2011). The cAMP/protein kinase a pathway and virulence in Cryptococcus neoformans. Mycobiology 39, 143-150. doi: 10.5941/MYCO.2011.39.3.143

Lee, N., D’Souza, C. A., and Kronstad, J. W. (2003). Of smuts, blasts, mildews, and blights: cAMP signaling in phytopathogenic fungi. Annu. Rev. Phytopathol. 41, 399-427. doi: 10.1146/annurev.phyto.41.052002.095728

Lengeler, K. B., Davidson, R. C., D'souza, C., Harashima, T., Shen, W. C., Wang, P., et al. (2000). Signal transduction cascades regulating fungal development and virulence. Microbiol. Mol. Biol. Rev. 64, 746-785. doi: 10.1128/MMBR.64.4.746-785.2000

Li, G., Zhou, X., and Xu, J. R. (2012). Genetic control of infection-related development in Magnaporthe oryzae. Curr. Opin. Microbiol. 15, 678-684. doi: 10.1016/j.mib.2012.09.004

Liu, L., Zhao, D., Zheng, L., Hsiang, T., Wei, Y., Fu, Y., et al. (2013). Identification of virulence genes in the crucifer anthracnose fungus Colletotrichum higginsianum by insertional mutagenesis. Microb. Pathog. 64, 6-17. doi: 10.1016/j.micpath.2013.06.001

Ma, L., Salas, O., Bowler, K., Oren-Young, L., Bar-Peled, M., and Sharon, A. (2017). Genetic alteration of UDP-rhamnose metabolism in Botrytis cinerea leads to accumulation of UDP-KDG that adversely affects development and pathogenicity. Mol. Plant Pathol. 18, 263-275. doi: 10.1111/mpp.12398

Martin-Urdiroz, M., Oses-Ruiz, M., Ryder, L. S., and Talbot, N. J. (2016). Investigating the biology of plant infection by the rice blast fungus Magnaporthe oryzae. Fungal Genet. Biol. 90, 61-68. doi: 10.1016/j.fgb.2015. 12.009

Mitchell, T. K., and Dean, R. A. (1995). The cAMP-dependent protein kinase catalytic subunit is required for appressorium formation and pathogenesis by the rice blast pathogen Magnaporthe grisea. Plant Cell 7, 1869-1878. doi: 10.1105/tpc.7.11.1869

Narusaka, Y., Narusaka, M., Park, P., Kubo, Y., Hirayama, T., Seki, M., et al. (2004). $\mathrm{RCH} 1$, a locus in Arabidopsis that confers resistance to the hemibiotrophic fungal pathogen Colletotrichum higginsianum. Mol. Plant Microbe Interact. 17, 749-762. doi: 10.1094/MPMI.2004.17.7.749

Ni, M., Rierson, S., Seo, J. A., and Yu, J. H. (2005). The $p k a B$ gene encoding the secondary protein kinase a catalytic subunit has a synthetic lethal interaction with pkaA and plays overlapping and opposite roles in Aspergillus nidulans. Eukaryot. Cell 4, 1465-1476. doi: 10.1128/EC.4.8.1465-1476.2005

O'Connell, R. J., Thon, M. R., Hacquard, S., Amyotte, S. G., Kleemann, J., Torres, M. F., et al. (2012). Lifestyle transitions in plant pathogenic Colletotrichum fungi deciphered by genome and transcriptome analyses. Nat. Genet. 44, 1060-1065. doi: $10.1038 / \mathrm{ng} .2372$

Oliver, B. G., Panepinto, J. C., Askew, D. S., and Rhodes, J. C. (2002). cAMP alteration of growth rate of Aspergillus fumigatus and Aspergillus niger is carbon-source dependent. Microbiology 148, 2627-2633. doi: 10.1099/00221287-148-8-2627

Pei-Bao, Z., Ren, A. Z., Xu, H. J., and Li, D. C. (2010). The gene $f p k 1$, encoding a cAMP-dependent protein kinase catalytic subunit homolog, is required for hyphal growth, spore germination, and plant infection in Fusarium verticillioides. J. Microbiol. Biotechnol. 20, 208-216. doi: $10.4014 / j m b .0905 .05044$ 
Ramanujam, R., and Naqvi, N. I. (2010). PdeH, a high-affinity cAMP phosphodiesterase, is a key regulator of asexual and pathogenic differentiation in Magnaporthe oryzae. PLoS Pathog. 6:e1000897. doi: 10.1371/journal.ppat.1000897

Robertson, L. S., and Fink, G. R. (1998). The three yeast A kinases have specific signaling functions in pseudohyphal growth. Proc. Natl. Acad. Sci. U.S.A. 95, 13783-13787. doi: 10.1073/pnas.95.23.13783

Schnell, N., Krems, B., and Entian, K. D. (1992). The PAR1(YAP1/SNQ3) gene of Saccharomyces cerevisiae, a c-jun homologue, is involved in oxygen metabolism. Curr. Genet. 21, 269-273. doi: 10.1007/BF00351681

Schumacher, J., Kokkelink, L., Huesmann, C., Jimenez-Teja, D., Collado, I. G., Barakat, R., et al. (2008). The cAMP dependent signaling pathway and its role in conidial germination, growth, and virulence of the gray mold Botrytis cinerea. Mol. Plant Microbe Interact. 21, 1443-1459. doi: 10.1094/MPMI-21-11-1443

Shimizu, K., and Keller, N. P. (2001). Genetic involvement of a cAMP dependent protein kinase in a g protein signaling pathway regulating morphological and chemical transitions in Aspergillus nidulans. Genetics 157, 591-600.

Takano, Y., Kikuchi, T., Kubo, Y., Hamer, J. E., Mise, K., and And Furusawa, I. (2000). The Colletotrichum lagenarium MAP kinase gene CMK1 regulates diverse aspects of fungal pathogenesis. Mol. Plant Microbe Interact. 13, 374-383. doi: 10.1094/MPMI.2000.13.4.374

Turrà, D., Segorbe, D., and Di Pietro, A. (2014). Protein kinases in plantpathogenic fungi: conserved regulators of infection. Annu. Rev. Phytopathol. 52, 267-288. doi: 10.1146/annurev-phyto-102313-050143

Wei, W., Xiong, Y., Zhu, W., Wang, N., Yang, G., and Peng, F. (2016). Colletotrichum higginsianum mitogen-activated protein kinase ChMK1: role in growth, cell wall integrity, colony melanization, and pathogenicity. Front. Microbiol. 7:1212. doi: 10.3389/fmicb.2016.01212

Xu, X., Wang, Y., Tian, C., and Liang, Y. (2016). The Colletotrichum gloeosporioides RhoB regulates cAMP and stress response pathways and is required for pathogenesis. Fungal Genet. Biol. 96, 12-24. doi: 10.1016/j.fgb.2016.09.002

Xue, C., Hsueh, Y. P., Chen, L., and Heitman, J. (2008). The RGS protein Crg2 regulates both pheromone and cAMP signalling in Cryptococcus neoformans. Mol. Microbiol. 70, 379-395. doi: 10.1111/j.1365-2958.2008.06417.x
Yamauchi, J., Takayanagi, N., Komeda, K., Takano, Y., and Okuno, T. (2004). cAMP-PKA signaling regulates multiple steps of fungal infection cooperatively with Cmk1 MAP kinase in Colletotrichum lagenarium. Mol. Plant Microbe Interact. 17, 1355-1365. doi: 10.1094/MPMI.2004.17.12.1355

Yang, K., Qin, Q., Liu, Y., Zhang, L., Liang, L., Lan, H., et al. (2016). Adenylate cyclase AcyA regulates development, aflatoxin biosynthesis and fungal virulence in Aspergillus flavus. Front. Cell. Infect. Microbiol. 6:190. doi: 10.3389/fcimb.2016.00190

Yang, X., Feng, H. V., and Yang, Y. S. (2008). Effects of silicon on flowering Chinese cabbage's anthracnose occurrence, flower stalk formation, and silicon uptake and accumulation. Chin. J. Appl. Ecol. 19, 1006-1012.

Yuan, Q., Chen, M., Yan, Y., Gu, Q., Huang, J., and Zheng, L. (2016). ChSte7 is required for vegetative growth and various plant infection processes in Colletotrichum higginsianum. Biomed Res. Int. 2016:7496569. doi: 10.1155/2016/7496569

Zhou, X., Zhang, H., Li, G., Shaw, B., and Xu, J. R. (2012). The Cyclaseassociated protein cap1 is important for proper regulation of infectionrelated morphogenesis in Magnaporthe oryzae. PLoS Pathog. 8:e1002911. doi: 10.1371/journal.ppat.1002911

Zhou, X., Zhao, X., Xue, C., Dai, Y., and Xu, J. R. (2014). Bypassing both surface attachment and surface recognition requirements for appressorium formation by overactive ras signaling in Magnaporthe oryzae. Mol. Plant Microbe Interact. 27, 996-1004. doi: 10.1094/MPMI-02-14-0052-R

Conflict of Interest Statement: The authors declare that the research was conducted in the absence of any commercial or financial relationships that could be construed as a potential conflict of interest.

Copyright (C) 2017 Zhu, Zhou, Xiong, Peng and Wei. This is an open-access article distributed under the terms of the Creative Commons Attribution License (CC BY). The use, distribution or reproduction in other forums is permitted, provided the original author(s) or licensor are credited and that the original publication in this journal is cited, in accordance with accepted academic practice. No use, distribution or reproduction is permitted which does not comply with these terms. 